\title{
Nonhydrogenic Effects in the Deceleration of Rydberg Atoms in Inhomogeneous Electric Fields
}

\author{
E. Vliegen, ${ }^{1}$ H. J. Wörner, ${ }^{1}$ T. P. Softley, ${ }^{2}$ and F. Merkt ${ }^{1, *}$ \\ ${ }^{1}$ Laboratorium für Physikalische Chemie, ETH Zürich CH-8093, Switzerland \\ ${ }^{2}$ Physical and Theoretical Chemistry Laboratory, South Parks Road, Oxford OXI 3QZ, United Kingdom
}

(Received 16 September 2003; published 23 January 2004)

Argon atoms in Stark states at $n \approx 25$ have been decelerated and accelerated in inhomogeneous electric fields. The acceleration and deceleration behavior can be understood only by considering the adiabatic Landau-Zener dynamics that take place at the avoided crossings between the Stark states and the limited fluorescence lifetimes of $\sim 10 \mu \mathrm{s}$.

DOI: 10.1103/PhysRevLett.92.033005

The production of cold molecules has been recently a topic of great interest; see [1] and references therein. Electrostatic deceleration has been successful in slowing down molecules with a permanent dipole moment in their ground state. To decelerate nonpolar particles a variation using the Stark effect in Rydberg states was first discussed in [2] and shown to be experimentally viable for $\mathrm{H}_{2}$ in [3].

The deceleration of $\mathrm{H}_{2}$ Rydberg molecules can be rationalized using an $\mathrm{H}$-atom-like model [3]. The energy levels of the $\mathrm{H}$ atom in an electric field $F$ are given, in atomic units, by

$$
E=I E-\frac{1}{2 n^{2}}+\frac{3}{2} n k F,
$$

where $I E \approx 1 / 2$ is the ionization energy, and $k$ is a quantum number which runs from $-\left(n-1-\left|m_{l}\right|\right)$ to $\left(n-1-\left|m_{l}\right|\right)$ in steps of two. Because the electrostatic potential is exactly Coulombic, the energy levels at zero field do not depend on $\ell$ and $m_{\ell}$ and are $n^{2}$ times degenerate. When an electric field is applied this degeneracy is lifted by the linear Stark effect [last term in Eq. (1)]. If an $\mathrm{H}$ atom is excited to a state the energy of which is lowered in an electric field ("redshifted" or "high-field seeking" state), it decelerates as it flies out of this field, whereas in a blueshifted, low-field seeking state, it accelerates. The loss/gain in kinetic energy is equal to $\Delta E=\frac{3}{2} n k \Delta F$. Because high Rydberg states $(n>10)$ of other atoms and molecules closely resemble those of the $\mathrm{H}$ atom, this method is, in principle, applicable to any particle in the gas phase. We show here that deviation from hydrogenic behavior can strongly affect the deceleration behavior. We discuss, with the example of the argon atom, the implications of (a) the deviation from a pure Coulomb potential which removes the degeneracy at zero field and induces avoided crossings in the Stark maps, (b) the adiabatic Landau-Zener dynamics that take place at these crossings, (c) the $m_{l} / m_{s}$ substructure, and (d) the finite lifetimes of the Rydberg states.

The experiment relies on the use of a tunable narrowbandwidth $\left(0.008 \mathrm{~cm}^{-1}\right)$ vacuum ultraviolet (VUV) laser
PACS numbers: $32.60 .+\mathrm{i}, 32.80 . \mathrm{Rm}, 34.60 .+\mathrm{z}, 39.90 .+\mathrm{d}$

[4] to excite Ar $n=15-30$ Rydberg states from the ${ }^{1} S_{0}$ ground state.

A skimmed supersonic expansion of the argon gas is crossed by the VUV laser beam at right angles in a region of homogeneous field (point $A$ in Fig. 1) or inhomogeneous field (point $B$ ). Setting the VUV polarization parallel (perpendicular) to the electric field accesses $M_{J}=0$ $\left(M_{J}=1\right)$ Stark states. The Rydberg atoms fly out of the photoexcitation region towards a microchannel plate (MCP) detector where an ionization signal is detected at a time corresponding to their time of flight (TOF). Spectra are obtained by monitoring the integrated TOF signal as a function of the VUV wave number. The spectral intensities reflect both the photoexcitation cross section and the decay of the excited states. Changes in the

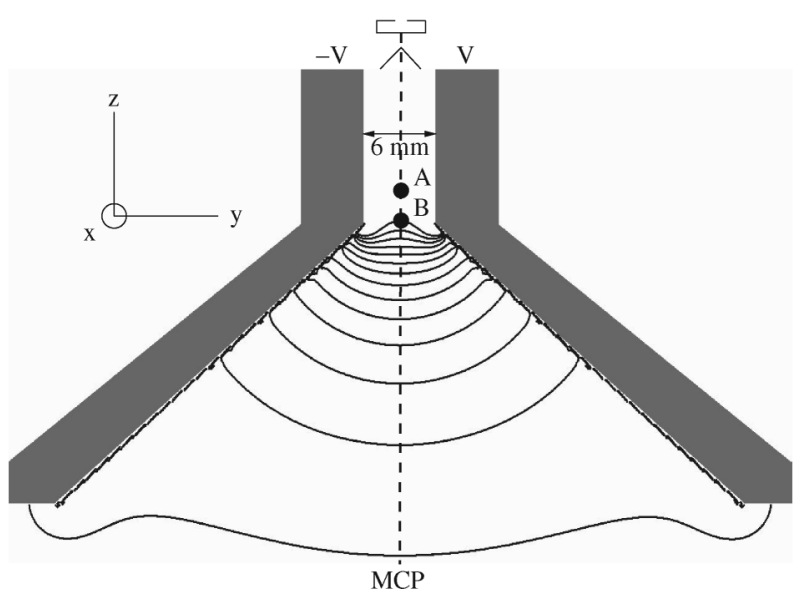

FIG. 1. Schematic overview of the experimental arrangement. The skimmed supersonic beam propagates in the $-z$ direction halfway between two metallic electrodes consisting of a plane parallel segment followed by a region in which the surface makes an angle of $45^{\circ}$ with the atomic beam. The VUV laser beam propagates parallel to the plate surfaces and perpendicular to the atomic beam. Photoexcitation is carried out in either homogeneous (point $A$ ) or inhomogeneous (point $B$ ) electric fields. The solid lines represent lines of constant electric fields ranging from $3 V / 2 \mathrm{~cm}^{-1}$ (top line) to $V / 5 \mathrm{~cm}^{-1}$ (bottom line) in steps of $V / 10 \mathrm{~cm}^{-1}$. 
quantum numbers of the Rydberg atoms can be observed by selective field ionization (SFI) [5]: pulsed voltages are applied to the electrodes and the resulting ions are deflected, leading to a depletion of the MCP signal.

Figure 2 shows a Stark map of the $M_{J}=0$ Rydberg states of $\mathrm{Ar}$ in the range around $n=22$ recorded at position $A$ in Fig. 1. At zero field, only the $n s$ and $n d$ states are observable. At increasing field, the manifold of high $\ell$ states, showing an H-like linear Stark effect, and the $p$ states become observable by Stark mixing with the $s$ and $d$ states. The $24 p[1 / 2]_{0}$ state ( $\mathrm{c}^{\prime}$ in Fig. 2) encounters the Stark manifold at $\sim 270 \mathrm{~V} / \mathrm{cm}$ and undergoes a series of avoided crossings with the most blueshifted states; see Fig. 3 below. Each $k$ state splits into several (up to four) fine-structure components corresponding to different ways of combining $m_{s}, m_{l}$, and $m_{j_{c}}$ to form $M_{J}=0$; see upper panel in Fig. 2. A striking aspect of Fig. 2 is the observation after $400 \mu$ s flight time of the $s$ and $d$ states, even though radiative lifetimes amount to $\sim 1 \mu \mathrm{s}$. This aspect will be discussed in more detail below.

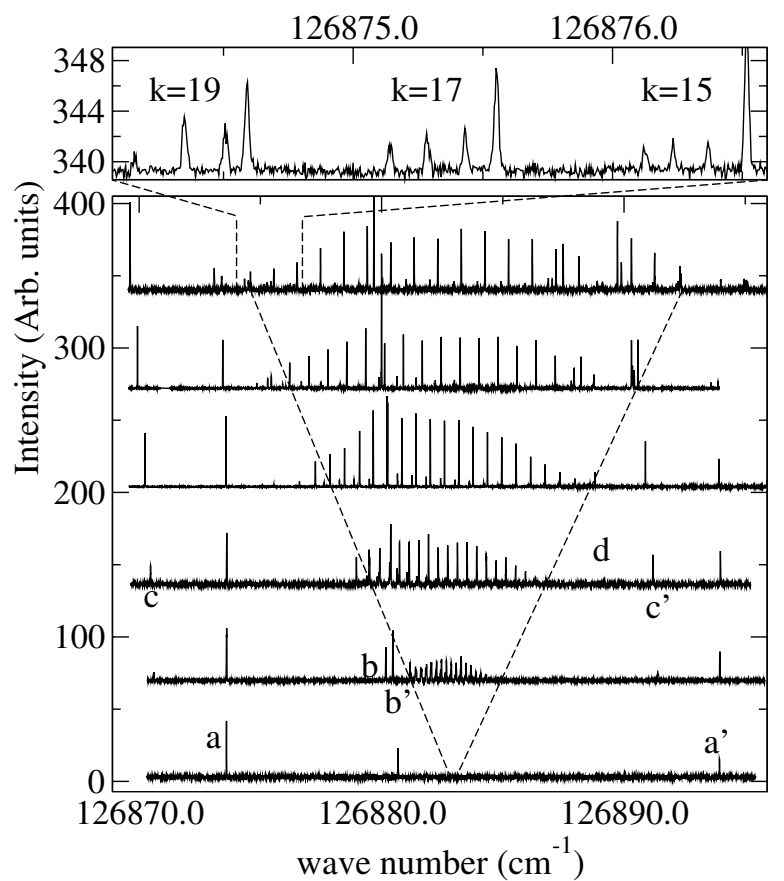

FIG. 2. Stark map of the $n=22, M_{J}=0$ argon Rydberg states. The origin of the intensity scale of each spectrum corresponds to the electric field strength in $\mathrm{V} / \mathrm{cm}$, from bottom to top $0,70,136,204,272,340 \mathrm{~V} / \mathrm{cm}$. The linear high $\ell$ Stark manifold is situated between the dotted lines. The states labeled $a, a^{\prime}, b, b^{\prime}, c, c^{\prime}$, and $d$ correspond to the $22 d[1 / 2]_{1}$, $23 d[1 / 2]_{1}, 22 d[3 / 2]_{1}, 24 s[3 / 2]_{1}, 23 p[1 / 2]_{0}, 24 p[1 / 2]_{0}$, and $24 p[5 / 2]_{2}$ states, respectively. The upper panel shows the fine structure of three red Stark states with $k=19,17$, and 15, from left to right, where $k$ represents a hydrogenic label determined using Eq. (1).
A detailed Stark map in the region of the avoided crossings between the $24 p[1 / 2]_{0}$ and the two bluest Stark states is displayed in Fig. 3. Each Stark state consists of four components, the upper three of which are closely spaced. The first set of avoided crossings involving the $24 p[1 / 2]_{0}$ state occurs with the four components of the $k=19$ Stark state at $F \approx 275 \mathrm{~V} / \mathrm{cm}$. Going from low to high field through this set of avoided crossings, one observes that the $24 p[1 / 2]_{0}$ state $(X)$ correlates adiabatically with the highest component of the $k=19$ Stark state $\left(Y^{\prime}\right)$, whereas it is the lowest component of the $k=19$ Stark state $(Y)$ that correlates with the $24 p[1 / 2]_{0}$ state $\left(X^{\prime}\right)$. The situation repeats itself when the $24 p[1 / 2]_{0}$ state encounters the four components of the next Stark state with $k=17$.

The inverted traces $d^{\prime}, e^{\prime}$, and $f^{\prime}$ in Fig. 3 correspond to measurements carried out by excitation at position $B$ in Fig. 1. Transitions to states with energies that vary rapidly with field strength are inhomogeneously broadened according to the behavior described in Ref. [6] and have a smaller peak intensity. States with a small field dependence, i.e., the $p$ states that undergo only a quadratic Stark effect (see trace $f^{\prime}$ ) and the Stark states at avoided crossings (see trace $e^{\prime}$ ), are hardly broadened with no significant decrease in peak intensity.

To quantify the role of the avoided crossings on the acceleration of Rydberg particles, the TOF distribution of the Rydberg atoms has been measured at the spectral positions and fields marked $X, X^{\prime}$ and $Y, Y^{\prime}$ in Fig. 3 following photoexcitation at point $B$ in Fig. 1. These positions correspond to excitation to the $24 p[1 / 2]_{0}$ state $\left(X\right.$ and $\left.X^{\prime}\right)$ and to the bluest Stark state $\left(Y\right.$ and $\left.Y^{\prime}\right)$ below and above the avoided crossing, respectively. No substantial acceleration is anticipated for the $p$ state because of the small deviation from its zero-field position, whereas an acceleration is expected for the bluest Stark state. This is

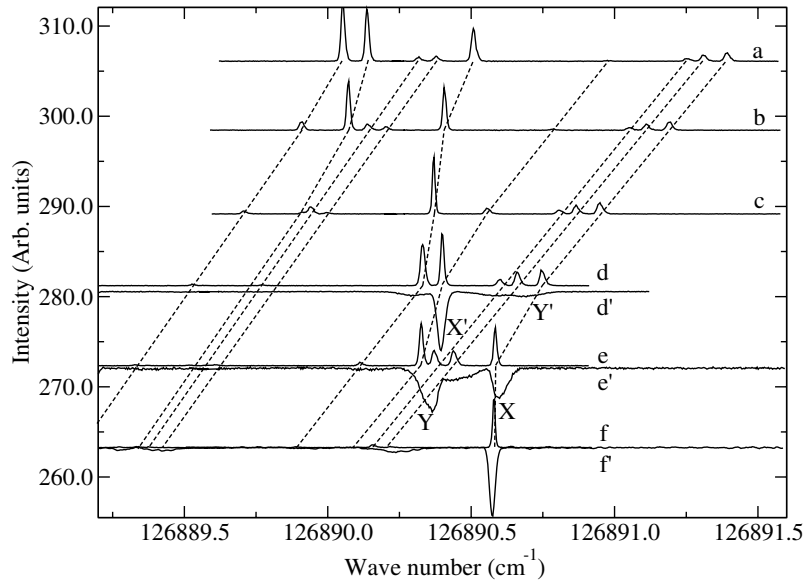

FIG. 3. Stark map of the avoided crossing of the $24 p[1 / 2]_{0}$ state with the high $\ell$ manifold. Traces pointing upward (downward) were measured in homogeneous (inhomogeneous) fields. The dashed lines show interpolations between measurements. 
indeed observed when excitation takes place at fields below the avoided crossing ( $X$ and $Y$ ) as illustrated in the upper panel in Fig. 4: the solid and dashed lines correspond to the TOF spectra measured following excitation to the $24 p[1 / 2]_{0}$ state $(X)$ and the highest component of the bluest Stark state $(Y)$, respectively. In the latter case, the decrease in the averaged TOF amounts to $1.3 \mu \mathrm{s}$, corresponding to an increase in velocity of $1.7 \mathrm{~m} / \mathrm{s}$ (i.e., $0.3 \%$ ) and in kinetic energy of $6.3 \times$ $10^{-23} \mathrm{~J}$ (i.e., $0.6 \%$ ). For reasons to be explained below, this acceleration amounts to only half of that expected on the basis of Eq. (1). Exactly the opposite behavior is observed when excitation occurs above the avoided crossing $\left(X^{\prime}\right.$ and $\left.Y^{\prime}\right)$; see lower panel in Fig. 4. In this case the $24 p[1 / 2]_{0}$ state is accelerated and the bluest Stark state does not change its velocity. This behavior indicates that the avoided crossing is traversed adiabatically when the atoms move towards lower field regions. Assuming a speed of $\sim 560 \mathrm{~m} / \mathrm{s}$, a minimum distance between the energy curves of $0.1 \mathrm{~cm}^{-1}$ at the avoided crossing, and an electric field gradient of $310 \mathrm{~V} / \mathrm{cm}^{2}$, we estimate from the Landau-Zener formula [7] that the probability of a diabatic passage is negligible. This result has profound implications for future deceleration experiments: the most shifted Stark states, which in the $\mathrm{H}$ atom would undergo the largest acceleration/deceleration, are precisely those that undergo avoided crossings with low $\ell$ states in all other atomic or molecular systems. The control of the translational motion of these states is correspondingly reduced. A similar explanation could be proposed for the less-than-expected deflection of the $n=$ $19, k=10$ component of krypton observed by Townsend et al. [8], which may be caused by avoided crossings with the $22 p$ states.

For a given Stark state the maximum deceleration can be achieved by maximizing the field strength and thus the

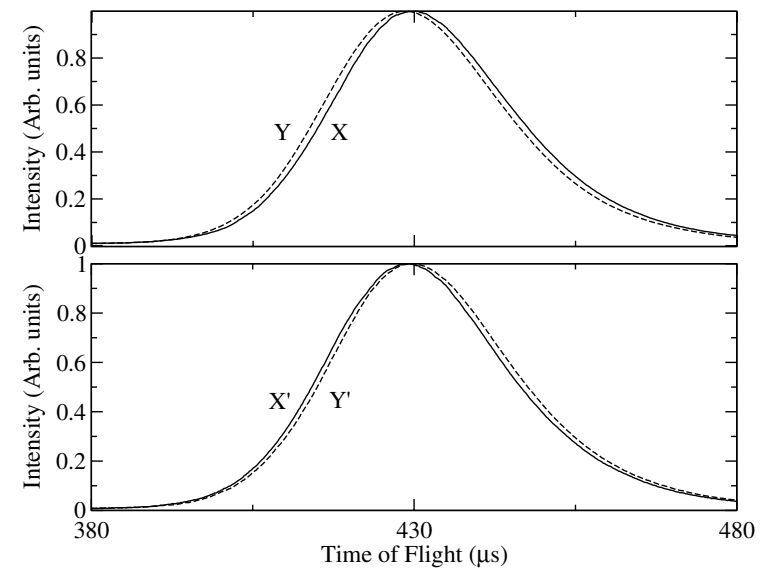

FIG. 4. TOF of the $24 p[1 / 2]_{0}$ (solid line) and the $n=22, k=$ 21 Stark state (dashed line) below (upper panel) and above (lower panel) the avoided crossing. The labels $X, Y, X^{\prime}$, and $Y^{\prime}$ refer to the spectral positions marked in Fig. 3. field inhomogeneity. An upper limit to the field strength is given by the field at the Inglis-Teller (IT) limit $\left(F_{\mathrm{IT}}=\right.$ $\left.1 / 3 n^{5}\right)$, because avoided crossings between Stark states of neighboring $n$ values above $F_{\text {IT }}$ lead to rapid changes of $k$ that are caused by adiabatic Landau-Zener dynamics. The most efficient way of influencing the velocity of the Rydberg particles consists of keeping the field strength at their instantaneous position as close to $F_{\text {IT }}$ as possible. This can be achieved by stepping up the voltage applied to the electrodes so as to compensate for the field changes along the trajectories. The Stark map of the $n=24$ and $n=25$ manifolds close to $F_{\mathrm{IT}}^{n=24}=215 \mathrm{~V} / \mathrm{cm}$ is displayed in Fig. 5. The second reddest Stark state of the $n=$ 25 manifold and the third bluest Stark state of the $n=24$ manifold (positions $b$ and $a$ in the lower panel in Fig. 5) were used in the experiment below because they have a larger intensity than the bluest and reddest states and the likelihood and effect of an avoided crossing with a low $\ell$ state are reduced. The electric field was stepped up during $10 \mu \mathrm{s}$ from an initial value of $205 \mathrm{~V} / \mathrm{cm}$ to a final value of $890 \mathrm{~V} / \mathrm{cm}$ in steps of $40 \mathrm{~V} / \mathrm{cm}$. The upper panel in Fig. 5 compares the TOF distributions with that obtained for the $25 d[1 / 2]_{1}$ state in the absence of an electric field. The velocity difference between the two sets of Rydberg atoms amounts to $1.25 \%$ of the initial velocity $(560 \mathrm{~m} / \mathrm{s})$ corresponding to a kinetic energy difference of $2.6 \times$ $10^{-22} \mathrm{~J}$, which, as in the previous example, is a factor of $\sim 2.5$ less than expected. Nevertheless, this velocity
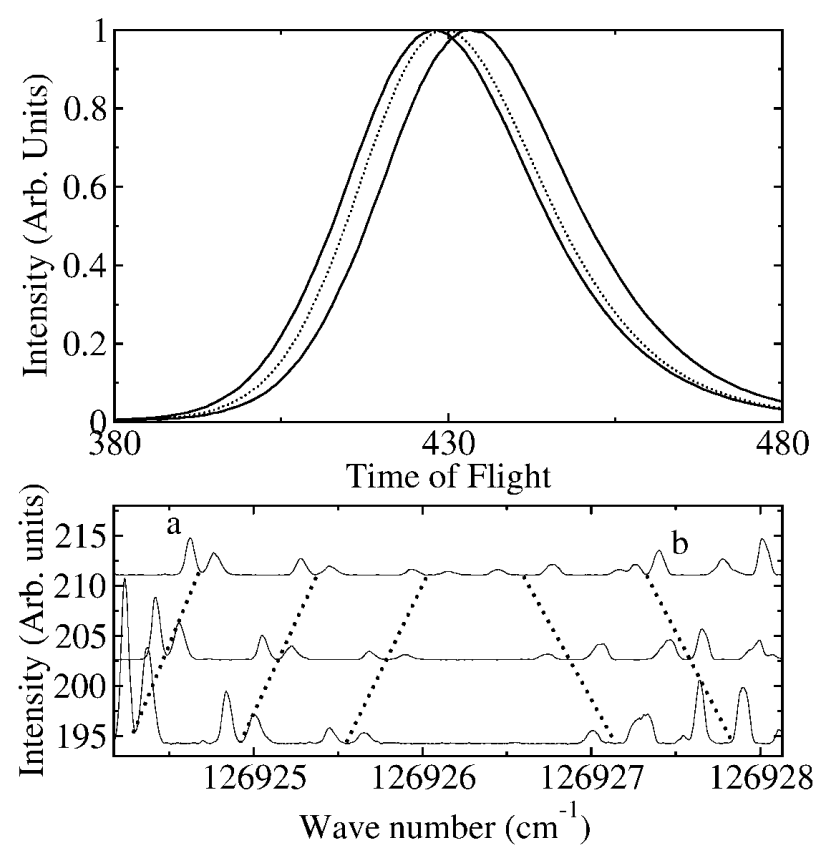

FIG. 5. Lower panel: Spectrum of the energy region where the $n=24$ and $n=25$ manifold meet. The dashed lines show the behavior of the three outer groups of Stark states in the $n=$ 24 manifold and the two outer groups of Stark states in the $n=$ 25 manifold. Upper panel: The TOF traces of state $a$ (left trace), state $b$ (right trace), and a zero-field state. 
difference is twice as large as obtained in a reference experiment carried out without stepping up the electric field. Moreover, TOF distributions in Fig. 5 indicate that the velocity of the blue state could not be controlled as efficiently as that of the red state. The reason for this behavior lies in an adiabatic traversal of an avoided crossing with a fine-structure component of the $p$ state.

The discrepancy of a factor of 2 between the magnitude of the expected and measured velocity changes originates primarily in the limited lifetime of the Rydberg states and the details of the decay dynamics. The importance of spontaneous emission and blackbody radiation [5] were discussed in Refs. [3,8], but could not be quantified experimentally. Figure 6 shows measurements which provide an explanation for the observation of a signal after $400 \mu$ s flight time and reconcile this observation with the lifetimes expected for Rydberg states around $n=20$ of, at most, a few microseconds. The dots in Fig. 6 represent the MCP signal obtained after initially preparing the $n=$ $25, k=16$ state and letting it fly for a well-defined delay time before turning the field off abruptly. Switching the field off immediately after photoexcitation leads to a signal about 3 times as large as when the field is not turned off. Turning off the field thus freezes the decay, presumably by locking the initial population into nondecaying states with high $\ell$ character [9]. The data can therefore be interpreted as a direct measurement of the decay by fluorescence of the Stark states to the ground state because all other states would have sufficient internal energy to be detected at the MCP. The fluorescence lifetime at $n \approx 25$ was measured to be $\sim 8 \mu \mathrm{s}$; this value is consistent with lifetimes of $\sim 1 \mu$ s measured by monitoring the fluorescence of the $s$ and $d$ states in this range of $n$, after taking into account the $\ell$ mixing induced by the field. We interpret the nondecaying part of the signal as being caused by $\ell$ and $m_{\ell}$ changing collisional processes as described in Refs. [10,11]. SFI measurements carried out at different delay times support this interpretation and show that such processes take place on a time scale of several microseconds and induce only small changes in $n(\Delta n<3)$. A similar behavior was observed for the zero-field $s$ and $d$ states. The role of the decay by blackbody-radiation-induced transitions cannot be assessed because the final states of the transitions have similar lifetimes to the initial states [5].

To conclude, we were successful in electrostatically decelerating a particle which is nonpolar in the ground state. The effect was about a factor of 2 smaller than expected on the basis of hydrogenic behavior because of adiabatic traversals of avoided crossings with low $\ell$ states and the limited lifetimes of the Rydberg atoms. To achieve maximal control of the translational motion of Rydberg particles, it is necessary to consider the nonhydrogenic effects in detail, including $m_{l} / m_{s}$ substructure and avoided crossings. The range of $n=20-30$ for

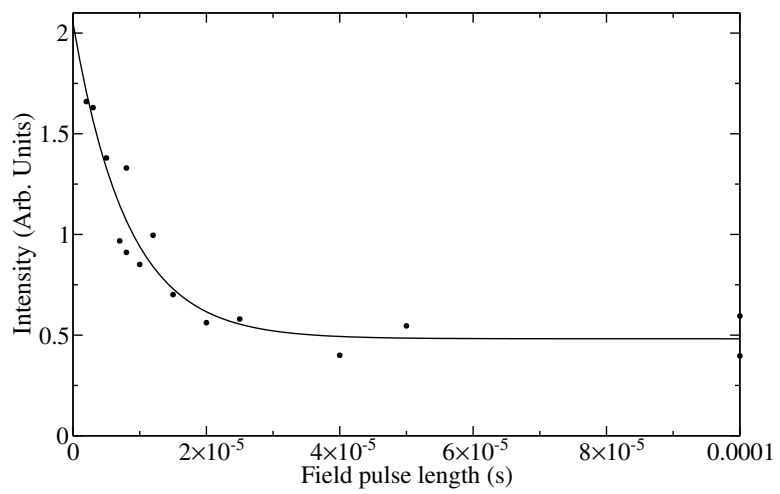

FIG. 6. Decay of the $n=25 k=16$ Stark state excited in a field of $200 \mathrm{~V} / \mathrm{cm}$.

such experiments is the optimal compromise between a sufficiently large Stark shift at the IT limit and sufficiently long lifetime. The measurement of the decay of the Rydberg states at $n=25$ sets a limit of $\sim 10 \mu$ s to the time available for deceleration. Stopping particles within $10 \mu$ s would necessitate a different electrode design. In order to decelerate light molecules to zero velocity in future experiments, it will be important to reduce the initial velocity. A kinetic energy loss of $\sim 1.8 \times 10^{-22} \mathrm{~J}$ was achieved for the $n=25, k=22$ state (Fig. 5). This energy corresponds in $\mathrm{H}_{2}$ to a velocity of $335 \mathrm{~m} / \mathrm{s}$, and such an initial velocity can be reached by seeding in a buffer gas or cooling the source.

This work is supported by the Swiss National Science Foundation, the ETH-Zürich, and by the Royal Society under Joint Project Grant No. 12832.

*Corresponding author.

[1] H. L. Bethlem and G. Meijer, Int. Rev. Phys. Chem. 22, 73 (2003).

[2] T. Breeden and H. Metcalf, Phys. Rev. Lett. 47, 1726 (1981).

[3] S. R. Procter, Y. Yamakita, F. Merkt, and T. P. Softley, Chem. Phys. Lett. 374, 667 (2003).

[4] U. Hollenstein, H. Palm, and F. Merkt, Rev. Sci. Instrum. 71, 4023 (2000).

[5] T. F. Gallagher, Rydberg Atoms (Cambridge University, Cambridge, 1994).

[6] A. Osterwalder and F. Merkt, Phys. Rev. Lett. 82, 1831 (1999).

[7] J. R. Rubbmark, M. M. Kash, M. G. Littman, and D. Kleppner, Phys. Rev. A 23, 3107 (1981).

[8] D. Townsend, A. L. Goodgame, S. R. Procter, S. R. Mackenzie, and T. P. Softley, J. Phys. B 34, 439 (2001).

[9] A. Held, L. Y. Baranov, H. L. Selzle, and E.W. Schlag, Chem. Phys. Lett. 291, 318 (1998).

[10] W. A. Chupka, J. Chem. Phys. 98, 4520 (1993).

[11] F. Merkt and R. N. Zare, J. Chem. Phys. 101, 3495 (1994). 